\title{
The MARCS model atmosphere code's first publication
}

\author{
F. Allard ${ }^{1,2}$ \\ 1 Centre de Recherche Astrophysique de Lyon, UMR 5574: CNRS, Université de Lyon, École Normale Supérieure de Lyon, \\ 46 allée d'Italie, 69364 Lyon Cedex 07, France \\ e-mail: fallard@ens-lyon.fr \\ 2 Institut d'Astrophysique de Paris, UMR 7095: CNRS, Université Pierre et Marie Curie-Paris 6, 98 bis boulevard Arago, 75014 Paris, \\ France
}

The article "A grid of model atmospheres for metal-deficient giant stars. I" by Gustafsson et al. (1975) received a normalization citation rate as high as 916 , i.e. one of the 40 most cited publications in A\&A. As one of the founding authors of another model atmosphere code - Phoenix in 1994 (Allard et al. 1994; Hauschildt et al. 1999, online), and focusing on the modeling of cooler, lower mass objects such as brown dwarfs and planets it is with, I hope, some impartiality and certainly great pleasure that I can describe the context of this article and its impact on the astrophysical community.

This publication was the very first presentation of the model atmosphere code $M A R C S$, a tool that came to dominate in the understanding of observations and the determination of stellar parameters (temperature scales, abundances, etc.) for decades. This article describes in such great detail the underlying assumptions, the algorithm, its implementation, and the resulting model structures of varying stellar parameters that, in his review for the Symposium "A Stellar Journey" in Uppsala, June 2008 celebrating the 65th birthday of Bengt Gustafsson (Lambert 2008, online), David Lambert stated "Construction of model atmospheres and the dependence of the atmospheres on various ingredients including line blanketing by metal and molecular (e.g. $\mathrm{CO}$ and $\mathrm{CN}$ ) lines are so clearly described that I recommend the paper as essential reading for all users of model atmospheres and spectrum synthesis codes. This paper and its 2008 derivative (Gustafsson et al. 2008) bracket my recommended reading list of Uppsala publications for students."

It describes the construction of a non-gray model atmosphere code in hydrostatic equilibrium, plane-parallel (PP) geometry, with convection included following the Henyey et al. (1965) formulation of the mixing length theory (MLT), and line opacities included in the form of an opacity distribution functions (ODF). Using this technique, models could be calculated with just a few hundred frequency points, and extensive grids for FGK stars could be calculated over a wider span of metallicity than could previously be achieved with similar non-gray models (Carbon \& Gingerich 1969; Parsons 1969; Alexander \& Johnson 1972; Johnson 1974; Peytremann 1970, 1974a,b, online). The ODFs were constructed with a program called SSG, originally devised for synthetic color calculations by Bell (Bell 1971). The line list used by this program was based on laboratory wavelengths and oscillator strengths, as well as "astrophysical oscil- lator strengths" derived from the solar spectrum. The total list contained about 50000 lines.

If this work had such a strong impact on the stellar astrophysics community, it is certainly because of the competence of the authors, their enthusiasm and sense of pragmatism in extracting the greatest potential from models, and their commitment to developing MARCS beyond assumptions and limitations whenever possible over the years, for example, by evolving from the ODF hypothesis to the more reliable opacity sampling (OS) method, to spherical geometry, to the account for departures from local thermodynamic equilibrium (LTE) and diffusion, to 3D hydrodynamical modeling. They motivated atomic and molecular physicists, via collaborations or by forming students and postdocs, in computing opacities of astrophysical interest. Today, modern simulations consider up to 85 million atomic and 1.2 billion molecular lines, using oscillator strengths derived by laboratory experiments in most cases. Many physical data are still missing, however, such as atomic collisional cross-sections for nonLTE applications (Barklem et al. 2008, online), e.g., the extrasolar planetary limbs (interactions with molecular hydrogen) observed in transits.

This development of MARCS was therefore also made possible by the parallel development of computational power that allows: i) computing of more complete and accurate opacities; ii) better accounting for these opacities in the model construction; iii) computing large model atmosphere grids spanning the range of stellar parameters and leading to more realistic interior and evolution models. The computation and calibration of color systems allowed the comparison of theory (e.g. isochrones) to observations in color-magnitude diagrams, a better understanding of the chemical evolution of the Galaxy (Edvardsson et al. 1993; Nordström et al. 2004, online), and the construction and improvement of galactic spectral synthesis simulations. The development of opacities and computational facilities allowed development of hydrodynamical models (Nordlund 1982), which are important in the determination of the velocity fields in these atmospheres and their impact on line broadening, the determination of abundances (Asplund et al. 2005), the determination of the mixing length (Ludwig et al. 2006), and finally the impact of dust cloud formation in late-type M dwarfs stars, brown dwarfs, and planets (Freytag et al. 2009). 
With extrasolar planets found around low-mass stars, the space missions GAIA, and PLATO profiling, the MARCS stellar model atmospheres and stellar astrophysics in general have wind in their sail for several decades ahead.

\section{References}

Alexander, D. R., \& Johnson, H. R. 1972, ApJ, 176, 629

Allard, F., Hauschildt, P. H., Miller, S., \& Tennyson, J. 1994, ApJ, 426, L39

Asplund, M., Grevesse, N., \& Sauval, A. J. 2005, in Cosmic Abundances as

Records of Stellar Evolution and Nucleosynthesis, ed. T. G. Barnes, III \& F. N. Bash, ASP Conf. Ser., 336, 25

Barklem, P. S., Korn, A. J., \& Plez, B. 2008, Phys. Scr. T, 133, 011001

Bell, R. A. 1971, MNRAS, 154, 343

Carbon, D. F., \& Gingerich, O. 1969, in Theory and Observation of Normal Stellar Atmospheres, ed. O. Gingerich, 377
Edvardsson, B., Andersen, J., Gustafsson, B., et al. 1993, A\&A, 275, 101 Freytag, B., Allard, F., Ludwig, H.-G., et al. 2009, in AIP Conf. Ser., ed. E. Stempels, 1094, 489

Gustafsson, B., Bell, R. A., Eriksson, K., \& Nordlund, A. 1975, A\&A, 42, 407 Gustafsson, B., Edvardsson, B., Eriksson, K., et al. 2008, A\&A, 486, 951 Hauschildt, P. H., Allard, F., \& Baron, E. 1999, ApJ, 512, 377

Henyey, L., Vardya, M. S., \& Bodenheimer, P. 1965, ApJ, 142, 841 Johnson, H. R. 1974, Model atmospheres for cool stars., ed. H. R. Johnson Lambert, D. L. 2008, Phys. Scr. T, 133, 014001

Ludwig, H.-G., Allard, F., \& Hauschildt, P. H. 2006, A\&A, 459, 599 Nordlund, A. 1982, A\&A, 107, 1

Nordström, B., Mayor, M., Andersen, J., et al. 2004, A\&A, 418, 989 Parsons, S. B. 1969, ApJS, 18, 127

Peytremann, E. 1970, Modèles d'atmospheres stellaires et interprétation de mesures photométriques, ed. E. Peytremann

Peytremann, E. 1974a, A\&A, 33, 203

Peytremann, E. 1974b, A\&AS, 18, 81 